\title{
Commentary: Metabolic syndrome and mortality
}

\author{
Andrew Farmer
}

Department of Primary Health Care, University of Oxford, Oxford OX3 7LF OX3 7LF senior research fellow andrew.farmer@ dphpc.ox.ac.uk
The metabolic syndrome was originally used to describe a clustering of risk factors for cardiovascular disease. It was hoped that study of metabolic syndrome might provide valuable information about the aetiology, epidemiology, treatment, and clinical prediction of diabetes and cardiovascular disease. Although most people with metabolic syndrome have insulin resistance, it is not known whether this is one of the primary causes. Further work has focused on the possibility of an association with an underlying inflammatory or prothrombotic state. The clinical value of diagnosing metabolic syndrome remains controversial.

A major attraction of categorising people by the presence of metabolic syndrome is the possibility of more accurate prediction of cardiovascular disease. Sundström and colleagues address this in their research. ${ }^{1}$ However, a recent review of prospective studies suggests that metabolic syndrome does only a modest job of predicting cardiovascular disease (estimated summary relative risk of 1.65 to 1.93 depending on definition of metabolic syndrome). ${ }^{2}$

Sundström and colleagues' approach is different. They evaluated the extent to which metabolic syndrome improves risk prediction beyond that achieved by conventional methods. ${ }^{1}$ They obtained improved estimates of risk of cardiovascular mortality ranging from 1.35 (95\% confidence interval 1.06 to 1.73$)$ to 1.59 (1.29 to 1.95 ) after taking into account smoking status, hypertension, serum cholesterol, and diabetes. ${ }^{1}$ Conventional risk factors to estimate risk of cardiovascular disease can be modelled in other ways, and at least three studies of this type have been identified, ${ }^{2}$ although each had fewer patients and was of shorter duration than the study by Sundström and colleagues. One combined metabolic syndrome with either the diabetes predicting model or the Framingham risk score and found no improvement in prediction of cardiovascular disease ${ }^{3}$; a second study also found no improvement on the Framingham risk score $^{4}$; and the third indicated that metabolic syndrome is associated with risk that is not entirely accounted for by the Framingham risk score.

In clinical practice, computer programs and charts derived from Framingham data are commonly used to establish risk of cardiovascular disease. Identifying people at high risk can target evidence based treatment, which has been shown in clinical outcome trials to substantially reduce the risk of cardiovascular disease. Limitations of these models are well recognised. In particular the models do not account for increased risk associated with raised (but not diagnostic of diabetes) levels of glucose.

Further work, building on current models for risk prediction and incorporating additional variables, is needed. For example, a coronary heart disease risk calculator for people with diabetes based on the United Kingdom prospective diabetes study data set has been developed to incorporate the duration and extent of glycaemia.

Should clinicians attempt to characterise people with the metabolic syndrome and use this information to determine possible cardiovascular risk? Younger people may not have a raised 10 year cardiovascular risk, but the use of identifying metabolic syndrome in this group is not clear. Joint British Society guidelines already suggest extending beyond conventional risk factors by including a family history of cardiovascular disease, central obesity, fasting glycaemia, and raised triglycerides. Unless further evidence emerges that metabolic syndrome confers a risk beyond the sum of the risk factors used in its definition, its use as a clinical predictor may be premature.

Competing interests: none declared.

1 Sundström J, Risérus U, Byberg L, Zethelius B, Lithell H, Lind L. Clinical value of the metabolic syndrome for long term prediction of total and : prospective, population based cohort study. BMJ 006 ; $329878-81$.

2 Ford FS. Risks for all-cause mortality, cardiovascular disease, and diabetes associated with the metabolic syndrome: a summary of the evidence. Diabetes Care 2005;28:1769-78.

3 Stern MP, Williams K, Gonzalez-Villalpando C, Hunt KJ, Haffner SM. Does the metabolic syndrome improve identification of individuals at risk of type 2 diabetes and/or cardiovascular disease? Diabetes Care 2004;27:2676-81.

4 McNeill AM, Rosamond WD, Girman CJ, Golden SH, Schmidt MI, East $\mathrm{HE}$, et al. The metabolic syndrome and 11-year risk of incident cardiovascular disease in the atherosclerosis risk in communities study. Diabetes Care 2005;28:385-90.

5 Girman CJ, Rhodes T, Mercuri M, Pyorala K, Kjekshus J, Pedersen TR, et al. The metabolic syndrome and risk of major coronary events in the al. The metabolic syndrome and risk of major coronary events in the coronary atherosclerosis prevention study. Am J Cardiol 2004;93:136-41. (Accepted 23 February 2006)

\section{Corrections and clarifications}

Paracetamol causes most liver failure in UK and US In this News article by Janice Tanne, published only on bmj.com, we mixed up our references and missed the key point, and these errors were compounded by an ambiguous title (bmj.com, 18 Mar 2006, News extra, doi:10.1136/ bmj.332.7542.628-a). We should have said that paracetamol (acetaminophen) overdose is a leading cause of acute liver failure in the United Kingdom and the United States. In the UK, though, most patients who get acute liver failure do so after intentional overdose whereas in the US nearly half of patients who get acute liver failure do so after unintentional overdose. And Robert Fontana describes himself as coauthor, not lead author. The studies reported in Hepatology (2005;42:1364-72) and Gastroenterology $(2006 ; 130: 687-94)$ were set in the US.

Tetraparesis associated with colchicine is probably due to inhibition by verapamil of the P-glycoprotein efflux pump in the blood-brain barrier

In this Drug Point by Uwe Tröger and colleagues we mislabelled two curves, and the error slipped through the proof stage (BMJ 2005;331:613, 17 Sep). The solid red line should indicate "norverapamil concentration in serum" and the broken orange line "colchicine concentration in cerebrospinal fluid." These labels were the wrong way round. 